\title{
Multi-commodity Flow Problem for Multi-hop Wireless Networks with Realistic Smart Antenna Model
}

\author{
Osama Bazan and Muhammad Jaseemuddin \\ Department of Electrical and Computer Engineering \\ Ryerson University, Toronto, Canada \\ \{obazan, jaseem\}@ee.ryerson.ca
}

\begin{abstract}
Utilizing smart antennas in multi-hop wireless networks can boost their performance. However, most of the work done in the context of wireless adhoc and sensor networking assume ideal or over-simplistic antenna patterns that overrate the performance benefits. In this paper, we compute the optimal throughput of multi-hop wireless networks with realistic smart antenna model. Our goal is to evaluate the network performance degradation using real smart antenna as compared to using the ideal model. We derive a generic interference model that can accommodate any antenna radiation pattern. We formulate the problem as a multi-commodity flow problem with novel interference constraints derived from our generic interference model. Our numerical results show that using a real-world smart antenna in a dense network results in up to $55 \%$ degradation in the throughput as compared to the case of ideal flat-topped antenna, whereas the throughput degradation is as much as $37 \%$ in a sparse network.
\end{abstract}

Keywords: Smart antennas, Multi-hop wireless networks, Optimization.

\section{Introduction}

Recently, the use of smart antennas in multi-hop wireless networks has received increasing attention in the research community due to their potential benefits over omni-directional antennas [1]. Smart antennas can increase the spatial reuse, extend the transmission range and reduce power consumption. Performance evaluations [1, 2, 3, 4, have demonstrated a tremendous improvement in the network performance. Most of the evaluations were performed using ideal or over-simplistic antenna patterns that overrate the performance benefits [3]. However, the results from experimental test-beds 4 have shown that realistic smart antennas still outperform the omni-directional antennas with a significant factor.

The pressing need for enhancing network capacity is a major motivation for using smart antennas. Interference is a dominant factor in limiting the capacity of wireless networks. By controlling the beamforming in a desired direction, 
interference in other directions is alleviated. This enables multiple simultaneous transmissions to happen in the same vicinity which increase the network capacity.

There have been several attempts to derive the optimal capacity bounds for multihop wireless networks. Gupta and Kumar [5] derived asymptotic capacity bounds. Jain et. al. [6] studied the impact of interference on multihop wireless network. They used the conflict graph to model interference. Kodialam et. al. 7] studied the joint routing and scheduling problem to achieve a given rate vector. In the context of smart antennas, Yi et. al. 8] extended the work in [5] to the case of ideal antennas. Spyropoulos et. al. 9 presented capacity bounds that are technology-based. Huang et. al. [10] formulated the problem as a single commodity flow problem in wireless sensor networks with directional antennas. However, they used a simple interference model and ideal antenna model. In [1], Peraki et. al. computed the maximum stable throughput in wireless networks with directional antennas by solving the minimum cut problem.

In this paper, we compute the optimal throughput of multi-hop wireless network using a realistic smart antenna model. Our goal is to evaluate the network performance degradation using real smart antenna model as compared to the ideal model. We formulate the problem as a multi-commodity flow problem. The contribution of this paper is two fold. First, we develop a generic interference model that can accommodate any antenna radiation pattern. Second, we formulate the multi-commodity problem with novel interference constraints. The differences between directional and omni-directional antennas impose the derivation of new interference constraints. We redefine the conflict graph [6] to take those differences into consideration.

In section 2 we provide basic antenna concepts. The generic interference model is derived in section 3 . We formulate the multi-commodity flow problem with the new interference constraints in section 4 . In section 5, we present the numerical results. We conclude the paper in section 6 .

\section{Preliminary}

In this section, we present few basic concepts related to smart antennas [12, 13. to introduce the terminology used to derive the interference model.

In contrast to the omni-directional antenna, the directional antenna radiates and receives energy from a direction more than the others. The gain of the antenna in any direction indicates the relative power in that direction to the power of omni-directional antenna. The peak gain over all directions is a major characteristic of any directional antenna. The antenna radiation pattern specifies the gain values in all directions of space. In this paper, we assume all nodes in the network to lie in a two-dimension plane, so, without the loss of generality, the gain of the antenna is a function of the azimuth angle only. A directional antenna pattern consists of a high gain main lobe and smaller gain side lobes. The axis of the main lobe is known as the boresight of the antenna. Another characteristic of a directional antenna is the half-power beamwidth which refers to the angle subtended by the directions on either side of the peak gain which 


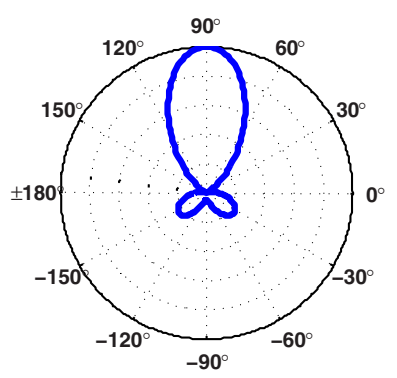

(a) Realistic directional antenna

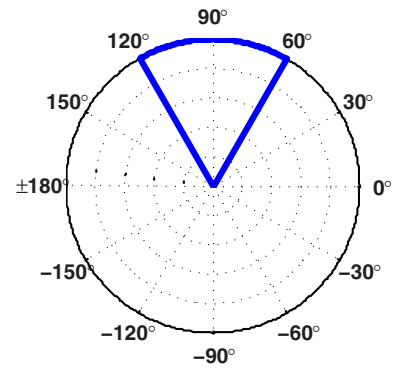

(b) Ideal antenna model

Fig. 1. Directional antenna radiation patterns

are $3 \mathrm{~dB}$ less in gain. An ideal antenna pattern has constant gain in main lobe and zero gain outside. Figure 1 shows antenna patterns for both realistic and ideal directional antennas. Since the transmission and reception characteristics on the antenna are reciprocal, the directional antenna has both transmission and reception gains.

According to Friss equation [13, the received power $P_{r}$ at a distance $r$ from a node with transmission power $P_{t}$ is:

$$
P_{r}=\frac{P_{t} G_{t}\left(\theta_{t}\right) G_{r}\left(\theta_{r}\right)}{K r^{\alpha}}
$$

where $G_{t}$ and $G_{r}$ are the transmitter and receiver gains, $\theta_{t}$ and $\theta_{r}$ are the transmitting and receiving angles measured with respect to the boresight, $\alpha$ is the path loss index and $K$ is a constant that depends on the wavelength. A receiver can receive the signal if the received power is greater than or equal to the receiver sensitivity threshold $\Omega$. Hence, the communication range $R$ in a certain direction is:

$$
R\left(\theta_{t}, \theta_{r}\right)=\left[\frac{P_{t} G_{t}\left(\theta_{t}\right) G_{r}\left(\theta_{r}\right)}{K \Omega}\right]^{1 / \alpha} .
$$

A smart beamforming antenna combines an antenna array with digital signal processing techniques to dynamically change its antenna pattern. Smart antennas can be classified into switched beam and steered beam systems. In switched beam systems, multiple fixed beam patterns are formed and the transceiver chooses the best among those beams. Hence, they do not guarantee maximum gain due to scalloping [13. Scalloping is the roll-off of the antenna pattern as a function of the angle from the boresight. In steered beam systems, the boresight of the main lobe can be directed in any direction. Additionally, nulls can be placed in the direction of interfering sources using sophisticated techniques. 


\section{The Interference Model}

In a wireless network, any transmission imposes an interference region in which other transmitters should not transmit to avoid collisions. The interference region generated by directional antenna transmissions is totally different than omnidirectional interference region since it depends not only on the distance between nodes but also on the boresights of the transmitting/receiving antennas and their radiation patterns. In this section, we present a generic interference protocol model to define the conditions for a successful wireless transmission.

Most of the previous work in adhoc networks with directional antennas [2,3,4, assume that a node transmits and receives in a directional mode but listens omnidirectionally when it is idle. We adopt this realistic assumption and hence the communication range will be function of $\theta_{t}$ only as $G_{r}=1$ in all directions,

$$
R\left(\theta_{t}\right)=\left[\frac{P_{t} G_{t}\left(\theta_{t}\right)}{K \Omega}\right]^{1 / \alpha} .
$$

A wireless link $(i, j)$ from node $i$ and node $j$ exists if

$$
d_{i j} \leq R_{i}\left(\theta_{i j}\right) \quad \frac{-\beta_{i}}{2} \leq \theta_{i j} \leq \frac{\beta_{i}}{2},
$$

where $d_{i j}$ is the distance between the nodes, $\theta_{i j}$ is the angle between the boresight of the antenna of node $i$ and the direction of $j$ and $\beta_{i}$ is the beamwidth of node $i$. Here, we assume that the wireless link exists only through the main lobe of the transmitter. Note that, equation (4) is generic to represent both switched beam and steered beam systems.

Since the interference may also occur through the side lobes, the interference range $R^{\prime}$ in any direction is given by

$$
R^{\prime}\left(\theta_{t}, \theta_{r}\right)=\Delta R\left(\theta_{t}, \theta_{r}\right),
$$

where $\Delta$ is greater than or equal to 1 and $R\left(\theta_{t}, \theta_{r}\right)$ is calculated as in (2).

In our interference model, both the sender and the receiver are required to be free of interference to reflect the case of most contention-based MAC protocols. For a successful transmission over existing wireless link $(i, j)$, any node $k(k \neq i$ or $j$ ) should avoid the communication in a certain direction if one of these conditions applies

$$
d_{k j} \leq R_{k}^{\prime}\left(\theta_{k j}, \theta_{j k}\right) \quad \text { or } \quad d_{k i} \leq R_{k}^{\prime}\left(\theta_{k i}, \theta_{i k}\right),
$$

where any angle $\theta_{x y}$ is subtended between the boresight of node $x$ and the direction of node $y$ and $\theta_{x y} \in[0,2 \pi)$. Note that, these conditions do not force node $k$ to always remain silent but allow it to communicate in non interfering directions. This introduces the spatial reuse factor into the interference model.

\section{Multi-commodity Flow Problem Formulation}

We are interested in the optimal throughput of multi-hop wireless networks with smart antennas. We formulate the problem as a multi-commodity flow 
problem with interference constraints. However, the complexity of a generic interference model demands novel interference constraints. Two major differences in our formulation distinguish it from [6]. First, we consider that the nodes may be equipped with different realistic antenna systems. We include the benefits of using smart antennas in our formulation. Second, we do not use the independent sets constraints that are computationally expensive and require global information. Instead, our constraints are localized in nature and can be implemented using distributed algorithms.

We consider a network graph $G(N, L)$ where $N$ is the set of nodes and $L$ is the set of directed wireless links. A directed link exists between two nodes if (4) is satisfied. Each node is equipped with real antenna system. Given a set of sourcedestination pairs, we assume that each source always has data to send and we are interested in the maximum total throughput the network can support. We assume the network operates in a time slotted mode, hence, the throughput we obtain will provide an upper bound for contention-based MAC protocols.

\subsection{The Conflict Graph}

We use the conflict graph to obtain the link capacity constraints due to interference. A conflict graph describes how the wireless link shares the medium. A vertex in the conflict graph is a link in the network graph while an edge in the conflict graph indicates that the two vertices (links in the network graph) interfere with each other. In [6], any two links that have a node in common are connected in the conflict graph. This is acceptable when omni-directional antennas are used. However, in the case of smart antennas, a node's link in one direction may not use the same wireless medium as its link in another direction and hence they should not have an edge in the conflict graph although they still cannot be active simultaneously due to the node capacity constraints. We modify the conflict graph to reflect the above argument. The main reason behind this modification is that interference from one direction should not affect the links on other directions. This is a major benefit of using smart beamforming antennas. In our modified conflict graph, there is an edge between two links with a common node if and only if they share the same wireless medium. In the case of switched beam systems, all the links transmitted/received by the same beam have edges in the conflict graph.

\subsection{The LP Formulation}

Given a wireless network $G(N, L)$ and a set of $M$ commodities each with sourcedestination pair $\left\{s^{m}, t^{m}\right\}$. We denote $x_{i j}^{m}$ as the amount of flow from the $m^{\text {th }}$ commodity over link $(i, j)$ normalized with respect to the capacity of the channel. $f^{m}$ denotes the flow coming out from source $s^{m}$. We denote Int $(i, j)$ as the set of links that interfere with link $(i, j)$ according to the conflict graph. The multicommodity flow problem can be formulated as the following LP formulation:

$$
\max \sum_{m \in M} f^{m}
$$


subject to

$$
\begin{gathered}
\sum_{(i, j) \in L} x_{i j}^{m}-\sum_{(j, i) \in L} x_{j i}^{m}=\left\{\begin{array}{ll}
f^{m} & i=s^{m} \\
0 & i=N \sim\left\{s^{m}, t^{m}\right\} \\
-f^{m} & i=t^{m}
\end{array} \quad \forall i, m,\right. \\
\sum_{m \in M} x_{i j}^{m}+\sum_{(p, q) \in \operatorname{Int}(i, j)} \sum_{m \in M} x_{p q}^{m} \leq 1 \quad \forall(i, j), \\
\sum_{m \in M}\left[\sum_{(i, j) \in L} x_{i j}^{m}+\sum_{(j, i) \in L} x_{j i}^{m}\right] \leq 1 \quad \forall i, \\
x_{i j}^{m} \geq 0
\end{gathered}
$$

The first constraint represents the flow conservation constraints at each node for each commodity. The second constraint is the link capacity constraint dictated by the interference model. The third constraint is the node capacity constraint in which the sum of the ingoing and outgoing flows should be less than the channel capacity. This is a necessary condition for scheduling feasibility 7 . Note that the node capacity constraint does not include any interference terms thanks to the beamforming antennas.

\section{$5 \quad$ Numerical Results}

In this section, we present some numerical results. Our goals are two fold: to evaluate how realistic antenna models impact the whole interference pattern of a multi-hop wireless network compared to their idealistic counterparts, and then to assess how this difference affects the optimal throughput of the network. We compare the resulting conflict graphs under different antenna models. Then, we compute the maximum flow by solving our multi-commodity flow problem using CPLEX. We study randomly deployed networks in $1500 \mathrm{~m}$ X $1500 \mathrm{~m}$ area. The link capacity is normalized to 1 . We assume two-ray propagation model. Each node is equipped with 6-beams switched beam smart antenna system with a peak gain of $10 \mathrm{dBi}$ and beamwidth of $60^{\circ}$. The maximum transmission range is $450 \mathrm{~m}$. We assume each node sends a flow to a random destination. We consider three different antenna patterns: (i) An ideal antenna pattern. (ii) A cone-plusball antenna model [1] which has an ideal main lobe in addition to low constant gain in all directions outside the main lobe averaging the side lobes. (iii) A real directional antenna pattern as the one shown in Fig. 11. This antenna pattern can be implemented using uniform circular antenna array with six isotropic elements with radius of 0.35 wavelength of the channel frequency [12].

Figure 2 shows the average total number of vertices in the conflict graph (directed links in the network graph) for different network densities. As expected, the network has less links when a realistic switched beam antenna pattern is used due to scalloping. Also, the difference between the number of ideal and realistic links increases in denser networks. Figure 3 shows the average vertex degree in the conflict graph. This number represents the average number of links 


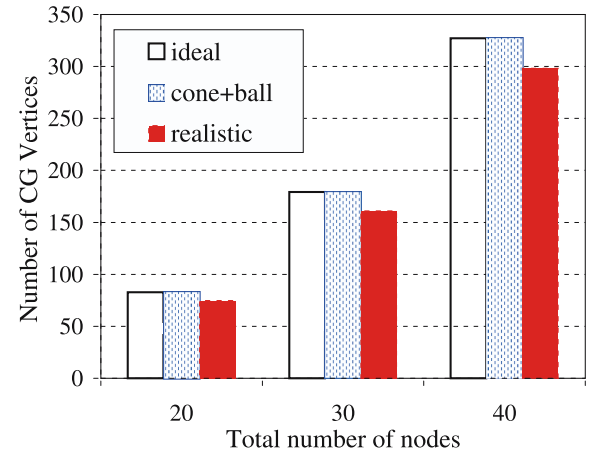

Fig. 2. Average number of conflict graph vertices with different node densities

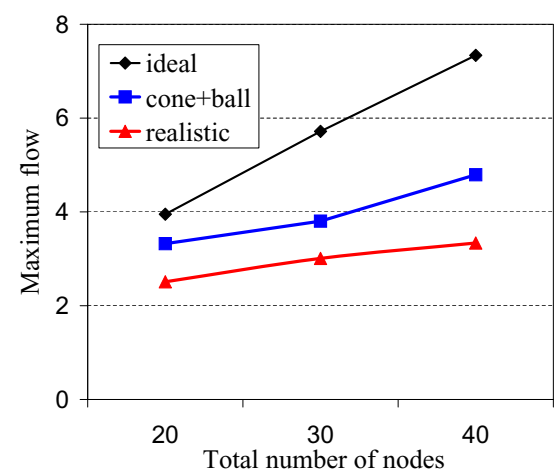

Fig. 4. Average maximum flow for different network densities

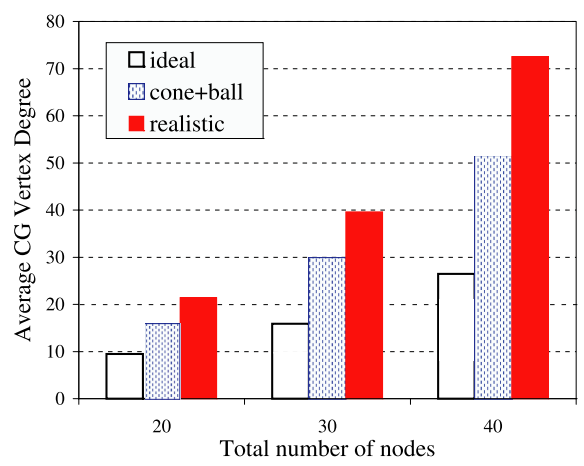

Fig. 3. Average vertex degree of the conflict graph with different node densities

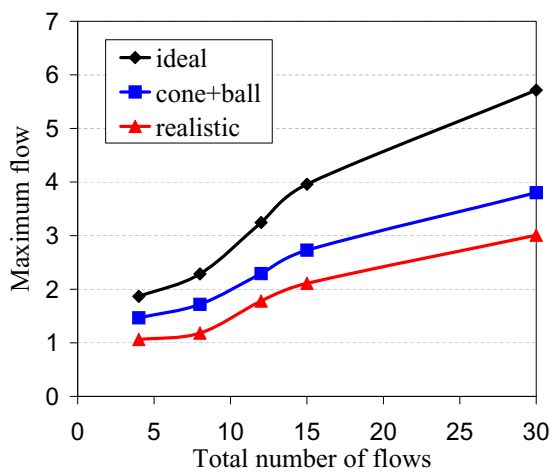

Fig. 5. Average maximum flow of 30-nodes network with different number of flows

interfering with each link. We can see that, in the case of 30-nodes network with a real antenna, the average vertex degree is almost 2.5 times the vertex degree in the case of ideal model. Figure 3 also shows that the average vertex degree of the conflict graph increases linearly with the density if an ideal antenna is assumed but increases exponentially if a real antenna is used. This implies that the effect of the side lobes is significant and should not be ignored.

Figure 4 shows the optimal throughput of random multi-hop wireless network with switched beam antennas for different network densities. It is clear that using realistic antenna patterns results in significant degradation in the throughput as opposed to the case of ideal antenna patterns. This is because of the significant difference in the interference pattern. In a sparse network (20 nodes), the realistic optimal throughput is $37 \%$ less than the ideal, whereas the realistic throughput degrades as much as $55 \%$ of the ideal in a dense network (40 nodes). Next, we consider solving the LP under different traffic loads. Figure 5 shows the average 
maximum flow for networks with 30 nodes and various numbers of flows. The results show that the percentage of performance degradation caused when a realistic antenna pattern is used is almost the same for different number of flows.

\section{Conclusions}

In this paper, we computed the optimal throughput of multi-hop wireless network with realistic smart antenna models. We presented a generic interference model to accommodate any antenna pattern. We formulated the problem as a multi-commodity flow problem with novel interference constraints. Our numerical results showed that using realistic antenna pattern could result in $55 \%$ degradation in the throughput as opposed to the ideal case. This implies that the effect of the side lobes is significant and should not be ignored. Our future work includes evaluating the performance improvement over omni-directional antennas and the design tradeoffs of using different smart antenna technologies.

\section{References}

1. Ramanathan, R.: On the Performance of Ad Hoc Networks with Beamforming Antennas. In: ACM MobiHoc, pp. 95-105 (2001)

2. Choudhury, R.R., Yang, X., Ramanathan, R., Vaidya, N.H.: On Designing MAC Protocols for Wireless Networks Using Directional Antennas. IEEE Transactions on Mobile Computing 5(5), 477-491 (2006)

3. Takai, M., Martin, J., Ren, A., Bagrodia, R.: Directional Virtual Carrier Sensing for Directional Antennas in Mobile Ad Hoc Networks. In: ACM MobiHoc, pp. 183-193 (2002)

4. Ramanathan, R., Redi, J., Santivanez, C., Wiggins, D., Polit, S.: Ad Hoc Networking with Directional Antennas: A Complete System Solution. In: IEEE WCNC, pp. $1-5(2006)$

5. Gupta, P., Kumar, P.R.: The Capacity of Wireless Networks. IEEE Transactions on Information Theory 46, 388-404 (2000)

6. Jain, K., Padhye, J., Padmanabhan, V., Qiu, L.: Impact of Interference on Multihop Wireless Network Performance. In: ACM MOBICOM, pp. 66-80 (2003)

7. Kodialam, M., Nandagopal, T.: Characterizing Achievable Rates in Multi-hop Wireless Networks: The Joint Routing and Scheduling Problem. In: ACM MOBICOM, pp. 42-54 (2003)

8. Yi, S., Pei, Y., Kalyanaraman, S.: On The Capacity Improvement of Ad Hoc Wireless Networks Using Directional Antennas. In: ACM MobiHoc, pp. 108-116 (2003)

9. Spyropoulos, A., Raghavendra, C.S.: Capacity Bounds for Ad-Hoc Networks Using Directional Antennas. In: IEEE ICC, pp. 348-352 (2003)

10. Huang, X., Wang, J., Fang, Y.: Achieving Maximum Flow in Interference-Aware Wireless Sensor Networks with Smart Antennas. Elsevier Ad Hoc Networks 5(6), 885-896 (2007)

11. Peraki, C., Servetto, S.D.: On the Maximum Stable Throughput Problem in Random Networks with Directional Antennas. In: ACM MobiHoc, pp. 76-87 (2003)

12. Balanis, C.A.: Antenna theory: Analysis and design. Wiley, Chichester (1997)

13. Liberti, J.C., Rappaport, T.S.: Smart Antennas for Wireless Communications. Prentice-Hall, NJ (1999) 